\title{
ON STRATIFIABLE AND ELASTIC SPACES
}

\author{
M. JEANNE HARRIS
}

(Communicated by Franklin D. Tall)

\begin{abstract}
We show that every stratifiable space has a $\sigma$-cushioned pair-base which is a function, and more generally, every linearly stratifiable space has a linearly cushioned pair-base which is a function. These results provide a correct proof for a theorem of Tamano and Vaughan.
\end{abstract}

\section{INTRODUCTION}

Elastic spaces were introduced by Tamano and Vaughan [TV] as a generalization of stratifiable spaces. Both kinds of spaces are defined in terms of a pair-base which has a cushion-type property. In [TV, Theorem 2], Tamano and Vaughan claim (1) every subspace of an elastic space is elastic, and (2) every stratifiable space is an elastic space. The proofs of these statements, however, are in doubt because of an oddity in the definition of elastic space: Tamano and Vaughan require that the pair-base not only have a cushion-type property but also be a function (or "map"). We call this an oddity because the definition of stratifiable space includes only a cushion-type property on the pair-base and does not require the pair-base to be a function. If one deletes the "function" requirement from the definition of Tamano and Vaughan, then the proof of [TV, Theorem 2] goes through. On the other hand, with the "function" property included in the definition of elastic, the proofs of both parts of [TV, Theorem 2] seem to be very nontrivial.

In this paper we show that every stratifiable space has a $\sigma$-cushioned pair-base which is a function. This is a new result for stratifiable spaces and, moreover, shows that part (2) of [TV, Theorem 2] is true using their definition of elastic space. More generally, it is an open problem whether the two definitions of elastic are equivalent.

First we recall some definitions, letting $X$ be a Hausdorff space throughout. A collection $\mathscr{P}=\left\{P \mid P=\left(P_{1}, P_{2}\right)\right\}$ of pairs of subsets of $X$ is a pair-base for $X$ if each $P_{1}$ is open and, for each element $x$ and each open set $U$ containing $x$, there is a pair $P$ in $\mathscr{P}$ for which $x \in P_{1} \subseteq P_{2} \subseteq U$. A collection $\mathscr{P}$ of pairs endowed with a linear order $\leq$ is linearly cushioned if, for each $\leq$-majorized

Received by the editors June 11, 1992 and, in revised form, February 15, 1993; presented June 18, 1992, Eighth Summer Conference on General Topology and Its Applications, Queens College, CUNY, New York.

1991 Mathematics Subject Classification. Primary 54E20.

Key words and phrases. Elastic space, linearly stratifiable. 
subcollection $\mathscr{P}^{\prime}$ (that is, each subcollection which has an upper bound in $\mathscr{P}$ with respect to $\leq), \mathrm{Cl}\left(\bigcup \mathscr{P}_{1}\right) \subseteq \bigcup \mathscr{P}_{2}$, where $\mathscr{P}_{j}=\left\{P_{j} \mid\left(P_{1}, P_{2}\right) \in \mathscr{P}\right\}$ for $j=1,2$. For infinite initial ordinal $\alpha$, the space $X$ is stratifiable over $\alpha$ $\left[\mathrm{V}_{2}\right]$, or linearly stratifiable, if there is a linearly cushioned pair-base $\mathscr{P}$ for $X$ and $\alpha$ is cofinal with $\mathscr{P}$. Equivalently $\left[\mathrm{V}_{2}\right]$, a space $(X, \mathfrak{I})$ is stratifiable over $\alpha$ iff there is a map $S: \alpha x \mathfrak{I} \rightarrow \mathfrak{I}$ which satisfies the following where we write $S_{\beta}(U)$ for $S(\beta, U):(1) \mathrm{Cl}\left(S_{\beta}(U)\right) \subseteq U$ for every $\beta<\alpha$ and every $U$ in $\mathfrak{I} ;(2) \bigcup\left\{S_{\beta}(U) \mid \beta<\alpha\right\}=U$ for every $U$ in $\mathfrak{I}$; (3) if $U \subseteq W$, then $S_{\beta}(U) \subseteq S_{\beta}(W)$ for every $\beta<\alpha$; and (4) if $\beta<\tau<\alpha$, then $S_{\beta}(U) \subseteq S_{\tau}(U)$ for every $U$ in $\mathfrak{I}$. Stratifiable spaces are those space which are stratifiable over $\omega$. Tamano and Vaughan [TV] introduced elastic spaces as a generalization of linearly stratifiable spaces, defining $X$ to be elastic iff $X$ has a pair-base $\mathscr{P}$ which, as a set of ordered pairs, is a function and an order $\sim$ defined on the set $\mathscr{P}_{1}=\left\{P_{1} \mid\left(P_{1}, P_{2}\right) \in \mathscr{P}\right\}$ such that (a) if $P_{1} \cap P_{1}^{\prime} \neq \varnothing$, then either $P_{1} \sim P_{1}^{\prime}$ or $P_{1}^{\prime} \sim P_{1} ;(\mathrm{b}) \sim$ is transitive; and (c) if $\mathscr{P}_{1}^{\prime}$ is a $\sim$-majorized subcollection of $\mathscr{P}_{1}$, then $\mathrm{Cl}\left(\bigcup\left\{P_{1} \mid P_{1} \in \mathscr{P}_{1}^{\prime}\right\}\right)$ is contained in $\bigcup\left\{P_{2} \mid\left(P_{1}, P_{2}\right) \in \mathscr{P}\right.$ and $P_{1} \in$ $\left.\mathscr{P}_{1}^{\prime}\right\}$.

Theorem 1. A linearly stratifiable space has a linearly cushioned pair-base that is a function.

Proof. Suppose $X$ is stratifiable over $\alpha$. Well order the points of $X$ as $\left\{x_{\mu} \mid \mu<\right.$ $\Theta\}$, and well order the open sets of $X$ as $\left\{O_{y} \mid \gamma\right\}$. Pair by pair we will choose for $X$ a pair-base $\mathscr{P}$ that is a function. At any time during the process of choosing the pairs, all pairs that have been chosen for $\mathscr{P}$ before that time will be referred to as "previously chosen pairs" and every set that is a coordinate of some previously chosen pair will be a "previously chosen set".

Step 1. First consider each open set $O_{\gamma}$ and, if some $\alpha$-stratification element $S_{\beta}\left(O_{\gamma}\right)$ of $O_{\gamma}$ is equal to $O_{\gamma}$, then choose the pair $\left(O_{\gamma}, O_{\gamma}\right)$ to belong to $\mathscr{P}$. Clearly, these pairs form a (possibly empty) function.

Step 2. For the first point $x_{\tau}$ at which there is an open set containing $x_{\tau}$ that contains no previously chosen open set that contains $x_{\tau}$, choose the first such open set $O_{\gamma(1)}$.

Step 3. Let $\mathscr{C}$ be the collection consisting of all $\alpha$-stratification elements $S_{\beta(1)}\left(O_{\gamma(1)}\right)$ of $O_{\gamma(1)}$, all $\alpha$-stratification elements $S_{\beta(2)}\left(S_{\beta(1)}\left(O_{\gamma(1)}\right)\right)$ of each $S_{\beta(1)}\left(O_{\gamma(1)}\right)$, all $\alpha$-stratification elements $S_{\beta(3)}\left(S_{\beta(2)}\left(S_{\beta(1)}\left(O_{\gamma(1)}\right)\right)\right.$ of each $S_{\beta(2)}\left(S_{\beta(1)}\left(O_{\gamma(1)}\right)\right)$, etc. Lexicographically order the collection of all possible arrays $(k, \beta(1), \beta(2), \beta(3), \ldots, \beta(k))$ where $k \in \mathbb{N}$ and $\beta(i)<\alpha$ for each $i$. For each element $C$ of $\mathscr{C}$ there is at least one array, and possibly many arrays, $(k, \beta(1), \beta(2), \beta(3), \ldots, \beta(k))$, for which $C$ is equal to the $\alpha$-stratification element $S_{\beta(k)}\left(\cdots\left(S_{\beta(3)}\left(S_{\beta(2)}\left(S_{\beta(1)}\left(O_{\gamma(1)}\right)\right)\right)\right)\right)$. With each $C$ in $\mathscr{C}$ associate the first such array and order the elements of $\mathscr{C}$ according to the lexicographic order of their associated arrays. This order $\sim$ will be a well order on $\mathscr{C}$.

The -least element of $\mathscr{C}$ that contains $x_{\tau}$ will be $S_{\beta^{\prime}(1)}\left(O_{\gamma(1)}\right)$ where $\beta^{\prime}(1)$ is the least ordinal less than $\alpha$ for which $x_{\tau}$ belongs to $S_{\beta^{\prime}(1)}\left(O_{\gamma(1)}\right)$. By choice of $O_{\gamma(1)}, S_{\beta^{\prime}(1)}\left(O_{\gamma(1)}\right)$ is not a previously chosen set; choose $\left(S_{\beta^{\prime}(1)}\left(O_{\gamma(1)}\right), O_{\gamma(1)}\right)$ to belong to $\mathscr{P}$. Continuing to consider the elements of $\mathscr{C}$ in order by $\sim$, for each $\beta>\beta^{\prime}(1)$ for which $S_{\beta}\left(O_{\gamma(1)}\right)$ is not a previously chosen set, choose the pair $\left(S_{\beta}\left(O_{\gamma(1)}\right), O_{\gamma(1)}\right)$ to belong to $\mathscr{P}$. After all $\alpha$-stratification elements 
$S_{\beta}\left(O_{\gamma(1)}\right)$ of $O_{\gamma(1)}$ have been considered, the $\sim$-least element of $\mathscr{C}$ that contains $x_{\tau}$ but is not previously chosen will be $S_{\beta^{\prime}(2)}\left(S_{\beta^{\prime}(1)}\left(O_{\gamma(1)}\right)\right)$ where $\beta^{\prime}(2)$ is the least ordinal for which $x_{\tau}$ belongs to $S_{\beta^{\prime}(2)}\left(S_{\beta^{\prime}(1)}\left(O_{\gamma(1)}\right)\right)$. By choice of $O_{\gamma(1)}$, the set $S_{\beta^{\prime}(2)}\left(S_{\beta^{\prime}(1)}\left(O_{\gamma(1)}\right)\right)$ can be neither $O_{\gamma(1)}$ nor $S_{\beta}\left(O_{\gamma(1)}\right)$ for $\beta \geq \beta^{\prime}(1)$. Choose the pair $\left(S_{\beta^{\prime}(2)}\left(S_{\beta^{\prime}(1)}\left(O_{\gamma(1)}\right)\right), S_{\beta^{\prime}(1)}\left(O_{\gamma(1)}\right)\right)$ to belong to $\mathscr{P}$ and, as above, for each $\beta>\beta^{\prime}(2)$, choose $\left(S_{\beta}\left(S_{\beta^{\prime}(1)}\left(O_{\gamma(1)}\right)\right), S_{\beta^{\prime}(1)}\left(O_{\gamma(1)}\right)\right)$ to belong to $\mathscr{P}$ if $S_{\beta}\left(S_{\beta^{\prime}(1)}\left(O_{\gamma(1)}\right)\right)$ is not a previously chosen set that contains $x_{\tau}$. Continue through $\mathscr{C}$, each time choosing the $\sim$-least element $C$ of $\mathscr{C}$ that contains $x_{\tau}$ but is not previously chosen. Each such $C$ is an $\alpha$-stratification element of some previously chosen element $C^{\prime}$ of $\mathscr{C}$; choose $\left(C, C^{\prime}\right)$ to belong to $\mathscr{P}$. Continue the process through the well-ordered set $\mathscr{C}$ or until a set $C$ is reached where all $\alpha$-stratification elements $S_{\beta}(C)$ have been previously chosen. The collection $\mathscr{P}$ is still a function because, when any pair is chosen for $\mathscr{P}$, its first coordinate is not a previously chosen set that contains $x_{\tau}$.

Step 4. If one exists, choose the first open set $O_{\gamma(2)}$ that contains the point $x_{\tau}$ but contains no previously chosen set that contains $x_{\tau}$. Repeat Step 3 on the collection of $\alpha$-stratification elements of $O_{\gamma(2)}, \alpha$-stratification elements of $\alpha$-stratificaton elements of $O_{\gamma(2)}$, etc.

Repeat Step 4, choosing open sets $O_{\gamma(\delta)}$ containing $x_{\tau}$ but no previously chosen set that contains $x_{\tau}$ and repeating Step 3 for each, until every open set that contains $x_{\tau}$ contains a previously chosen set that contains $x_{\tau}$. All the pairs that have been chosen from the beginning of Step 2 to this point and all coordinates of those pairs are said to have been chosen for $x_{\tau}$.

Step 5. If the collection of previously chosen pairs does not form a pair-base for $X$, let $x_{\mu}$ be the first point of $X$ at which this collection does not contain a local pair-base. Repeat Steps 2 and 3 for $x_{\mu}$ and then repeat Step 4 for $x_{\mu}$ until every open set that contains $x_{\mu}$ contains a previously chosen set that contains $x_{\mu}$.

Now the collection $\mathscr{P}$ of all previously chosen pairs is a function, because when a pair is included in $\mathscr{P}$ its first coordinate must not be previously chosen and by the following $\mathscr{P}$ is a pair-base for $X$. If element $x$ of $X$ belongs to open set $U$, then $U$ must contain a previously chosen set that contains $x$, for otherwise Step 5 would have been repeated at $x$. Since the order in which the previously chosen sets were chosen is a well order, let $W$ be the first previously chosen set such that $x \in W \subseteq U$. If $(W, W)$ does not belong to $\mathscr{P}$, suppose $W$ was chosen for the point $x_{\mu}$. Let $\zeta<\alpha$ be the first ordinal such that $S_{\zeta}(W)$ contains both $x$ and $x_{\mu}$. Since $S_{\zeta}(W)$ is not equal to $W$, then $S_{\zeta}(W)$ cannot have been chosen before $W$ by choice of $W$. Now either (a) $W$ is one of the sets $O$ chosen in Step 2 or at the beginning of Step 4 or (b) $W$ is of the form $S_{\beta(k)}\left(S_{\beta(k-1)}\left(\cdots\left(S_{\beta(2)}\left(S_{\beta(1)}(O)\right)\right) \cdots\right)\right)$ for some set $O$ and some array $(k, \beta(1), \beta(2), \beta(3), \ldots, \beta(k))$. In case $(a)$, any set chosen after $W$ but before $S_{\zeta}(W)$ must have the form $S_{\beta}(W)$ with $\beta<\zeta$ and, not containing both $x$ and $x_{\mu}$, cannot be equal to $S_{\zeta}(W)$. In case (b), a set $V$ chosen after $W$ is chosen but before $S_{\zeta}(W)$ is considered must have either (1) the form $S_{\beta}\left(S_{\beta(k-1)}\left(\cdots\left(S_{\beta(2)}\left(S_{\beta(1)}(O)\right)\right) \cdots\right)\right)$ for some $\beta>\beta(k)$ or (2) the form $S_{\beta}(W)$ for some $\beta<\zeta$. In case (1), the fact that $V$ is chosen implies that $W$ is a proper subset of $V$ so that $S_{\zeta}(W)$ is not equal to $V$. In case (2), if $V=S_{\beta}(W)$ with $\beta<\zeta$ then, as in case (a), $V$ could not contain both $x$ and $x_{\mu}$ and could not be equal to $S_{\zeta}(W)$. This meansthat, at the time when 
the $\alpha$-stratification elements of $W$ were being considered with respect to $x_{\mu}$, the set $S_{\zeta}(W)$ was not previously chosen and the pair $\left(S_{\zeta}(W), W\right)$ was chosen then to belong to $\mathscr{P}$. Thus $x \in S_{\zeta}(W) \subseteq W \subseteq U$ and $\left(S_{\zeta}(W), W\right)$ belongs to $\mathscr{P}$, and $\mathscr{P}$ is a pair-base for $X$.

Next we need a linear order on $\mathscr{P}$; we will use an order analogous to that described in the proof of Theorem 2 [TV]. Every pair of $\mathscr{P}$ has the form $\left(P_{1}, P_{2}\right)$ where $P_{1}$ is $S_{\beta}\left(P_{2}\right)$ for some $\beta<\alpha$. For each $\beta<\alpha$, let $\mathscr{P}_{\beta}=$ $\left\{\left(P_{1}, P_{2}\right) \in \mathscr{P} \mid P_{1}=S_{\beta}\left(P_{2}\right)\right\}$ and let $<_{\beta}$ be a well-order for $\mathscr{P}_{\beta}$. Order $\mathscr{P}$ by $\leq$ where $P \leq P^{\prime}$ for $P$ and $P^{\prime}$ in $\mathscr{P}$ iff $P \in \mathscr{P}_{\beta}, P^{\prime} \in \mathscr{P}_{\beta^{\prime}}$ and either $\beta<\beta^{\prime}$ or $\left(\beta=\beta^{\prime}\right.$ and $\left.P<\beta P^{\prime}\right)$. Then $\leq$ is a linear order on $\mathscr{P}$. If $\mathscr{P}^{\prime}$ is a $\leq$-majorized subcollection of $\mathscr{P}$, then, for some ordinal $\beta^{*}<\alpha, \mathscr{P}^{\prime}$ is a subset of $\bigcup_{\beta<\beta^{*}} \mathscr{P}_{\beta}$. Then

$$
\begin{aligned}
\mathrm{Cl}\left[\bigcup\left(\mathscr{P}^{\prime}\right)_{1}\right] & =\mathrm{Cl}\left[\bigcup_{\beta \leq \beta^{*}}\left\{P_{1} \mid\left(P_{1}, P_{2}\right) \in \mathscr{P}^{\prime} \cap \mathscr{P}_{\beta}\right\}\right] \\
& =\mathrm{Cl}\left[\bigcup_{\beta \leq \beta^{*}}\left\{S_{\beta}\left(P_{2}\right) \mid\left(P_{1}, P_{2}\right) \in \mathscr{P}^{\prime} \cap \mathscr{P}_{\beta}\right\}\right] \\
& \subseteq \mathrm{Cl}\left[\bigcup_{\beta \leq \beta^{*}} S_{\beta}\left(\bigcup\left\{P_{2} \mid\left(P_{1}, P_{2}\right) \in \mathscr{P}^{\prime} \cap \mathscr{P}_{\beta}\right\}\right)\right] \\
& \subseteq \mathrm{Cl}\left[\bigcup_{\beta \leq \beta^{*}} S_{\beta^{*}}\left(\bigcup\left\{P_{2} \mid\left(P_{1}, P_{2}\right) \in \mathscr{P}^{\prime} \cap \mathscr{P}_{\beta}\right\}\right)\right] \\
& \subseteq \mathrm{Cl}\left[S_{\beta^{*}}\left(\bigcup\left(\mathscr{P}^{\prime}\right)_{2}\right)\right] \subseteq \bigcup\left(\mathscr{P}^{\prime}\right)_{2} .
\end{aligned}
$$

Thus $\mathscr{P}$ is linearly cushioned. Since the collection $\mathscr{P}$ of pairs of open sets is a function, $\mathscr{P}$ is the desired pair-base.

Since $\mathscr{P}$ being a linearly cushioned pair-base with $\omega$ cofinal with $\mathscr{P}$ is equivalent to $\mathscr{P}$ being a $\sigma$-cushioned pair-base, we have the following result for stratifiable spaces.

Corollary 2. A stratifiable space has a $\sigma$-cushioned pair-base that is a function.

Corollary 3. A linearly stratifiable space is elastic.

Proof. By Theorem 1, suppose a linearly stratifiable space $X$ has a linearly cushioned pair-base $\mathscr{P}$ which is a function. Using the given linear order $\leq$ on $\mathscr{P}$, define $\sim$ on $\mathscr{P}_{1}$ by $P_{1} \sim P_{1}^{\prime}$ iff $\left(P_{1}, P_{2}\right) \leq\left(P_{1}^{\prime}, P_{2}^{\prime}\right)$. Since $\mathscr{P}$ is a function, $\sim$ is a linear order on $\mathscr{P}_{1}$ and therefore satisfies $(a)$ and $(b)$ of the definition of an elastic space. If $\mathscr{P}_{1}^{\prime}$ is a subcollection of $\mathscr{P}_{1} \sim$-majorized by $P_{1}^{\prime \prime}$, then $\left\{P \in \mathscr{P} \mid P_{1} \in \mathscr{P}_{1}^{\prime}\right\}$ is a subcollection of $\mathscr{P} \leq$-majorized by $\left(P_{1}^{\prime \prime}, P_{2}^{\prime \prime}\right)$ and $\mathrm{Cl}\left(\bigcup \mathscr{P}_{1}^{\prime}\right) \subseteq \bigcup P_{2}^{\prime}$. Thus, $(\mathscr{P}, \sim)$ is an elastic base for $X$.

Corollary 4. A stratifiable space is elastic.

ACKNOWLEDGMENT

The author is grateful to Professors Robert Heath and Jerry Vaughan for all their advice. 


\section{BIBLIOGRAPHY}

[Bo1] C. J. R. Borges, Elastic spaces and related concepts, Kobe J. Math. 5 (1988), 311-316.

[Bo2] _ Four generalizations of stratifiable spaces, General Topology, Proc. Third Prague Topology Sympos., 1971, pp. 73-76.

[G2] G. Gruenhage, The Sorgenfrey line is not elastic, Proc. Amer. Math. Soc. 38 (1973), 665-666.

[Mo] P. J. Moody, Neighborhood conditions on topological spaces, Ph.D. thesis, Oxford, 1989.

[P] J. Pope, Some results concerning elastic spaces, Ph.D. thesis, University of North Carolina at Chapel Hill, 1972.

[TV] T. Tamano and J. Vaughan, Paracompactness and elastic spaces, Proc. Amer. Math. Soc. 28 (1971), 299-303.

[V1] J. E. Vaughan, Linearly ordered collections and paracompactness, Proc. Amer. Math. Soc. 24 (1970), 186-192.

[V2] _ Linearly stratifiable spaces, Pacific J. Math. 43 (1972), 253-266.

Department of Mathematics, Fairmont State College, Fairmont, West Virginia 26554

E-mail address: mjhef scvax.wrnet.edu 